procedure has the potential to improve issues with colonoscopy capacity and diagnostic workforce in the NHS, with a larger scale study now needed. We have previously published on the training requirements and learning curve for the novel colonoscopy procedure and will also present data on computer aided analysis and interpretation of findings at colonoscopy.

\section{AWE-02 MORPHOLOGICAL AND MOLECULAR MARKERS FOR COEXISTENT ADENOCARCINOMA IN LOW-GRADE DYSPLASTIC AREAS OF HIGH-GRADE COLORECTAL ADENOMAS}

Andrew Emmanuel*, Salvador Diaz-Cano, Shraddha Gulati, Savvas Papagrigoriadis, Bu Hayee, Mr Amyn Haji. King's College Hospital NHS Foundtaion Trust, London, UK

\subsection{6/gutjnl-2019-BSGAbstracts.20}

Introduction Successful endoscopic resection (ER) relies on endoscopic diagnosis to predict the risk of invasive cancer. However, a detailed evaluation of histopathological features and the molecular profile of the dysplastic mucosa to predict coexistent invasive cancer is not available.

Methods ER of large colorectal adenomas (2011-2016) were analysed. A subset containing high-grade dysplasia, intramucosal cancer or invasive cancer was identified and subjected to detailed histopathological analysis: ulceration, distribution of high-grade dysplasia, dysplastic nuclear grade, presence/distribution of necrosis, and distribution of tumour-infiltrating lymphocytes (TIL). Microdissection, DNA extraction and nextgeneration sequencing using a human clinically relevant tumour panel of 24 genes were performed separately for two areas with the highest morphological grade from each lesion.

Results ER was performed for 418 large $(\geq 20 \mathrm{~mm})$ adenomas. Histopathological genetic evaluation was available in 70 high grade cases. Coexistent adenocarcinoma significantly correlated with adenomatous mucosa featuring ulceration, mixed interface/interstitial TIL, multifocal high nuclear grade, infiltrative edges, and multifocal intraluminal necrosis. Multifocal intraluminal necrosis and high nuclear grade in the adjacent lowgrade dysplastic mucosa were driven by cooperative genetic abnormalities of high-impact (FLT4), moderate impact (KRAS/ NRAS for infiltrative edges, FLT4, TP53, ERBB2), and low impact (FGFR3, PDGFA).

Conclusions The dysplastic stage of high-grade adenomas is characterized by multiple cooperative genetic mutations. A subset of these identify a risk of coexistent adenocarcinoma with a close correlation between genetic markers of angiogenesis (FLT4), receptor activation (RAS/ERBB2), genome maintenance (TP53) and stromal reaction (FGFR3, PDGFRA) with morphological features defined by high nuclear grade, intraluminal necrosis, and inflammatory stromal reaction.

\section{AWE-03 MANAGEMENT OF PANCREATIC WALLED OFF NECROSIS WITH LUMEN APPOSING METAL STENTS: UK SINGLE CENTRE EXPERIENCE}

George Goodchild*, Holly Theaker, Jonathan Potts, Harry Martin, Javed Iqbal, Michael Chapman, Stephen Pereira, George Webster, Gavin Johnson. UCLH, London, UK

10.1136/gutjnl-2019-BSGAbstracts.21

Background Endoscopic ultrasound (EUS)-guided transmural drainage is the preferred treatment for symptomatic pancreatic walled-off necrosis (WON). Lumen apposing metal stents (LAMS) are increasingly used, although their advantage over plastic stents remains unclear. Placement of a plastic pigtail stent within the LAMS may help maintain patency and help avoid the need for subsequent endoscopic necrosectomy. We aim to describe the success and complication rates for the use of LAMS in the management of WON.

Methods All patients undergoing EUS-guided cystgastrostomy using LAMS for WON between July 2015-January 2019 were included. Data collected included procedural technique, rates of technical and clinical success and complications. All patients had up to date CT or MR scans and all were reviewed in the specialist MDM prior to intervention.

Results 40 patients underwent LAMS placement for WON, $75 \%$ male, median age 53 years (range 23-79). The aetiology of pancreatitis was: gallstones $(n=17)$, alcohol $(n=12)$, idiopathic $(n=9)$ and post-ERCP $(n=2)$. Mean collection size on pre-procedure imaging was $12 \mathrm{~cm}$ (range 4-22). 21 $(52.5 \%)$ procedures were performed under general anaesthesia, $14(35 \%)$ propofol sedation and 5 (12.5\%) conscious sedation. LAMS were placed from the stomach $(n=38,95 \%)$ or duodenum $(n=2,5 \%)$ and were successfully deployed in 39 (98\%). LAMS diameter was $8 \mathrm{~mm}(\mathrm{n}=7), 10 \mathrm{~mm}(\mathrm{n}=5)$, $12 \mathrm{~mm}(\mathrm{n}=1), 15 \mathrm{~mm}(\mathrm{n}=24)$ and $20 \mathrm{~mm}(\mathrm{n}=3)$. Pigtail stents were placed within the LAMS in 14 cases $(35 \%)$ but did not significantly alter the need for subsequent endoscopic necrosectomy (28\% with pigtail stent vs $27 \%$ without). Follow up CT demonstrated a reduction in collection size in all cases with a mean maximal diameter of $2.6 \mathrm{~cm}$ $(0-5 \mathrm{~cm})$.

Successful drainage was achieved in $98 \%$ at 3 months (1 patient died from complications of acute pancreatitis, unrelated to stent placement) and no patient required percutaneous or surgical drainage. There were two complications; 1) late migration of the gastric flange into the collection, presenting with sepsis 6 weeks post insertion and successfully retrieved following placement of a second LAMS and 2) buried gastric flange, leading to delayed LAMS removal and successfully retrieved 7 months post insertion after transmural tract dilation using large diameter biopsy forceps. Median time to LAMS removal was 51 days (range 26-80). LAMS remained in situ for 21 months in one patient lost to follow-up but was removed without complication.

Conclusion Transmural drainage of pancreatic WON using LAMS has excellent rates of technical and clinical success with few complications. Unlike in other published series, we did not observe any late bleeding complications. In our cohort, placement of a pigtail stent through the LAMS did not negate the need for subsequent endoscopic necrosectomy.

\section{AWE-04 NEAR-FOCUS NBI CLASSIFICATION OF VILLOUS ATROPHY IN SUSPECTED COELIAC DISEASE: INTERNATIONAL DEVELOPMENT AND VALIDATION}

\footnotetext{
${ }^{1}$ Shraddha Gulati* ${ }^{*}{ }^{1}$ Andrew Emmanuel, ${ }^{1}$ Mehul Patel, ${ }^{2}$ Tareq El-Menabawey, ${ }^{3}$ Zuzana Vackova, ${ }^{1}$ Polychronis Pavlidis, ${ }^{1}$ Nishmi Gunasingam, ${ }^{6}$ Vivienne Sayer, ${ }^{2}$ Alberto Murino, ${ }^{1}$ Patrick Dubois, ${ }^{4}$ Helmut Neumann, ${ }^{3}$ Jan Martinek, ${ }^{5}$ Amrita Sethi, ${ }^{1}$ Amyn Haji, 'Bu'Hussain Hayee. 'King's College Hospital, London, UK; ${ }^{2}$ Royal Free Hoapital, London, UK; ${ }^{3}$ IKEM, Prague, Czech Republic; ' University Medical Center, Mainz, Germany; ${ }^{5}$ NewYork-Presbyterian, New York, USA; ${ }^{6}$ Princess Royal University Hospital, London, UK
}

10.1136/gutjnl-2019-BSGAbstracts.22 
Introduction There are no agreed endoscopic signs for the diagnosis of villous atrophy(VA) in coeliac disease(CD), necessitating biopsies and for both diagnosis and exclusion. Here we evaluated the role of near focus Narrow Band Imaging (NF-NBI) for the assessment of villous architecture in suspected $\mathrm{CD}$ with development and further validation of a novel NF-NBI classification.

Methods Patients with symptoms/investigations warranting duodenal biopsy were prospectively recruited between September 2017 to August 2018. Six paired NF-white light(NFWLE) and NF-NBI images with biopsy (2 from the first part of the duodenum,4 from the second) were obtained from each patient. Histopathology grading used Marsh-Oberhuber classification(M-O). Images were reviewed for quality and biopsy orientation. Separate images were used for development of the classification, training and validation steps. A modified Delphi process was performed on images and video recordings by 3 endoscopists to define NF-NBI characteristics (included if kappa>0.6). 13 blinded endoscopists(5 expert, 8 non-expert) underwent a short training module on the proposed NBI classification and evaluated paired(NF-WLE/NFNBI) images.

Results 100 consecutive patients were recruited and $n=97$ completed the study $(66 \mathrm{~F}, 51.2 \pm 17.3 \mathrm{yrs})$. TTG positive $\mathrm{n}=17 / 89$. Prevalence of $\mathrm{M}-\mathrm{O} \mathrm{VA}(3 \mathrm{a} / 3 \mathrm{~b} / 3 \mathrm{c})$ in D1 and D2 biopsies was 52/194(27\%) and 70/388(18\%) respectively. After image quality and biopsy orientation review; 548 paired images remained. 498 paired images developed the classification; 3 descriptors:Villous shape, vascular discrimination, crypt phenotype proposed the classification. 13 endoscopists evaluated 50 paired images each(D1:20, M-O $0: 26$, M-O 3a-3b:13, M-O 3c:11). Pooled diagnostic test summary statistics(\%) for NF-NBI diagnosis of VA(Subtotal/ total atrophy) were: Sensitivity 97.9(91.67-100), specificity 82.15(62.5-100), NPV 97.7(92.59-100) and accuracy 89.7 (80-96) respectively. Mean difference in confidence using NF-NBI vs NF-WLE significantly improved when assessing the first part duodenum: The classification was further validated in histopathologically proven duodenitis $(n=15)$ images with no features of VA using the proposed classification.

Conclusion A novel NF-NBI classification for VA had been validated to reliably diagnose VA in suspected CD amongst both expert/non-expert endoscopists using readily available equipment and required only short training supporting translation to wider practice.

\section{AWE-05 CASE SERIES OF PER-ORAL ENDOSCOPIC PYLOROMYOTOMY IN PATIENTS WITH REFRACTORY GASTROPARESIS: A SINGLE CENTRE EXPERIENCE}

Nishmi Gunasingam*, Mehul Patel, Shradda Gulati, Amyn Haji, Bu'Hussain Hayee. King's College Hospital NHS Foundation Trust, London, UK

\subsection{6/gutjnl-2019-BSGAbstracts.23}

Introduction Gastroparesis (GP) is a disorder evidenced by delayed gastric emptying in the absence of mechanical obstruction, commonly idiopathic or secondary to diabetes mellitus. G-POEM has been used to treat refractory gastroparesis and we present our initial experience with this novel endoscopic technique.
Methods All patients undergoing G-POEM for refractory GP from May 2018 onwards were included, with data extracted from the hospital electronic patient record. Procedures were performed by one endoscopists at our centre with experience in submucosal endoscopy. Efficacy at 3 months was assessed by reduction of symptom score (Gastroparesis Cardinal Symptom Index; GCSI), with secondary considerations including technical success, procedural complications, hospital length of stay and hospitalisations after treatment.

Results 10 patients (10F; mean age $40.3 \pm 15.4$ y) were included (5 diabetic, 5 idiopathic). Mean duration of disease was $9.5 \pm 5.6 \mathrm{y}$, with 4 months median duration of follow-up post-procedure. 6 patients had previous treatment with botulinum toxin, and one gastric electrical stimulator. Technical success was achieved in all cases while, at 3 months, 8 had improvement total GCSI (mean scores 3.978 vs 2.076 $(\mathrm{p}=0.008))$ and each GCSI subtype score: nausea and vomiting $(3.63$ vs $1.70, \mathrm{p}=0.012)$; fullness $(4.30$ vs 2.30 , $\mathrm{p}=0.012$ ); bloating (4 vs $2.22, \mathrm{p}=0.28$ ). Mean hospital stay was $9 \pm 13 \mathrm{~d}$, with 5 patients staying less than 4 days and two with prolonged admission. One significant adverse event was recorded: abdominal collection around the myotomy site requiring a prolonged hospital admission of 18 days for intravenous antibiotics in one patient.

Only one patient required hospitalisation for gastroparesis symptoms after their G-POEM procedure (this was the same patient who did not achieve clinical success with reduction in their GCSI scores).

Conclusion G-POEM is a promising therapeutic treatment for patients with refractory GP, with significant improvement in symptoms and, in our cohort a dramatic reduction in the need for hospitalisation in short-term follow up. A European sham-controlled study is under way and longer-term data are required to confidently determine its role in the management of such a challenging condition.

\section{AWE-06 OUTCOMES OF HEMOSPRAY USE IN TUMOUR RELATED UPPER GASTROINTESTINAL BLEEDS: OUTCOMES FROM THE HEMOSPRAY REGISTRY}

${ }^{1}$ Mohamed Hussein*, ${ }^{2}$ Durayd Alzoubaidi, ${ }^{3}$ Miguel Fraile, ${ }^{3}$ Jacobo Ortiz Fernandez-sordo ${ }^{3}$ Krish Ragunath, ${ }^{4}$ Radu Rusu, ${ }^{4}$ Jason Dunn, ${ }^{14}$ Johannes Rey, ${ }^{5}$ Shraddha Gulati, ${ }^{5}$ Bu'Hussain Hayee, ${ }^{6}$ Selena Dixon, ${ }^{6}$ Sulleman Moreea, ${ }^{7}$ Duncan Napier, ${ }^{7}$ John Anderson, ${ }^{8}$ Martin Dahan, ${ }^{9}$ Max Hu, ${ }^{9}$ Patricia Duarte, ${ }^{9}$ Phil Boger, ${ }^{10}$ John Mcgoran, ${ }^{10}$ Inder Mainie, ${ }^{11}$ Alberto Murino, ${ }^{11}$ Sina Jameie-oskooei, ${ }^{11}$ Edward Despott, ${ }^{12}$ Cora Steinheber, ${ }^{12}$ Martin Goetz, ${ }^{13}$ Sharmila Subramaniam, ${ }^{13}$ Pradeep Bhandari, ${ }^{1,2}$ Laurence Lovat, ${ }^{8}$ Emmanuel Coron, ${ }^{14}$ Ralf Kiesslich, ${ }^{1}$ Rehan Haidry. ${ }^{1} U C L H, U K ;{ }^{2} U C L, U K ;{ }^{3}$ Nottingham Digestive Diseases Centre, UK; ${ }^{4}$ Guys and St Thomas Hospitals, UK; ${ }^{5}$ Kings college Hospital, UK; ${ }^{6}$ Bradford Teaching Hospital NHS Trust, UK; ${ }^{7}$ Gloustershire Hospitals NHS Foundation Trust, UK; ${ }^{8}$ University Hospital of Nantes, France; ${ }^{9}$ University Hospital Southampton, UK; ${ }^{10}$ Belfast NHS Trust, UK; ${ }^{11}$ Royal Free Hospital, UK; ${ }^{12}$ Tübingen University Hospital, UK; ${ }^{13}$ University of Portsmouth, UK; ${ }^{14}$ Horst Schmidt Kliniken, UK

\subsection{6/gutjnl-2019-BSGAbstracts.24}

Introduction Patients with tumour related upper gastrointestinal bleeds (UGIBs) are challenging to treat. Hemospray (Cook Medical, North Carolina, USA) is a novel haemostatic powder for GI bleeding. The primary aim was to look at outcomes of UGIBs secondary to tumours who had Hemospray therapy in 13 centres.

Methods Data was prospectively collected on the use of hemospray from specialist centres in the UK, France, Germany (Jan'16-September'18). Hemospray was used for UGIBs 